\title{
Revealing the therapeutic targets and molecular mechanisms of emodin-treated coronavirus disease 2019 via a systematic study of network pharmacology
}

\author{
Hai-Xia Du ${ }^{1,{ }^{*}}$, Jia-Qi Zhu ${ }^{2,}{ }^{*}$, Jing Chen ${ }^{2}$, Hui-Fen Zhou², Jie-Hong Yang ${ }^{1}$, Hai-Tong Wan ${ }^{1,2}$ \\ ${ }^{1}$ College of Basic Medical Science, Zhejiang Chinese Medical University, Hangzhou 310053, China \\ ${ }^{2}$ College of Life Science, Zhejiang Chinese Medical University, Hangzhou 310053, China \\ "Equal contribution and Co-first authors
}

Correspondence to: Jie-Hong Yang, Hai-Tong Wan; email: yjhong@zcmu.edu.cn, wanhaitong@zcmu.edu.cn

Keywords: emodin, COVID-19, pathway, network pharmacology, molecular docking

Received: December 30, 2020 Accepted: May 13, $2021 \quad$ Published: June 4, 2021

Copyright: (C) 2021 Du et al. This is an open access article distributed under the terms of the Creative Commons Attribution License (CC BY 3.0), which permits unrestricted use, distribution, and reproduction in any medium, provided the original author and source are credited.

\section{ABSTRACT}

Emodin has shown pharmacological effects in the treatment of infection with severe acute respiratory syndrome coronavirus-2, which leads to coronavirus disease 2019 (COVID-19). Thus, we speculated that emodin may possess anti-COVID-19 activity. In this study, using bioinformatics databases, we screened and harvested the candidate genes or targets of emodin and COVID-19 prior to the determination of pharmacological targets and molecular mechanisms of emodin against COVID-19. We discovered core targets for the treatment of COVID-19, including mitogen-activated protein kinase 1 (MAPK1), tumor protein (TP53), tumor necrosis factor (TNF), caspase-3 (CASP3), epidermal growth factor receptor (EGFR), vascular endothelial growth factor A (VEGFA), interleukin 1B (IL1B), mitogen-activated protein kinase 14 (MAPK14), prostaglandinendoperoxide synthase 2 (PTGS2), B-cell lymphoma-2-like protein 1 (BCL2L1), interleukin-8 (CXCL8), myeloid cell leukemia-1 (MCL1), and colony stimulating factor 2 (CSF2). The GO analysis of emodin against COVID-19 mainly included cytokine-mediated signaling pathway, response to lipopolysaccharide, response to molecule of bacterial origin, developmental process involved in reproduction, and reproductive structure development. The KEGG results exhibited that the molecular pathways mainly included IL-17 signaling pathway, AGE-RAGE signaling pathway in diabetic complications, TNF signaling pathway, pertussis, proteoglycans in cancer, pathways in cancer, MAPK signaling pathway, NOD-like receptor signaling pathway, NF-kappa B signaling pathway, etc. Also, molecular docking results revealed the docking capability between emodin and COVID-19 and the potential pharmacological activity of emodin against COVID-19. Taken together, these findings uncovered the targets and pharmacological mechanisms of emodin for treating COVID-19 and suggested that the vital targets might be used as biomarkers against COVID-19.

\section{INTRODUCTION}

The etiology of coronavirus disease 2019 (COVID-19) is caused by infection with severe acute respiratory syndrome coronavirus 2 (SARS-CoV-2), with patients experiencing fever, dry cough, and fatigue as common symptoms [1]. In comparison with other coronaviruses, SARS-CoV-2 shows lower pathogenesis but higher transmission capacity [2, 3]. To date, COVID-19 has been sweeping through many different geographical areas in a timescale of minutes and seconds $[4,5]$. Indeed, the worldwide outbreak of the disease has emerged as a severe threat to public health, claiming many lives, and has come to be 
regarded as a global health emergency in January 2020 and a pandemic in March 2020, as declared by the World Health Organization (WHO) [6]. In light of these declarations and especially given the novelty of this condition, exploring and developing effective therapeutic drugs or vaccines is crucial, yet effective therapeutics are still limited in number even one year later.

Traditional Chinese medicine (TCM) has been trialed to treat COVID-19 and has achieved good curative effects [7]. Emodin (1, 3, 8-trihydroxy-6methylanthraquinone) is an anthraquinone derivative from the roots and barks of Rheum palmatum, Polygonum cuspidatum and Polygonum multiflorum [8, 9], and has evident pharmacological benefits, supporting the inhibition of hepatitis B virus replication in vitro or in vivo [10], the anti-proliferation of lung cancer cells [11], and the regulation of immune response or inflammatory activities [12]. Previous works have reported on the inhibition of influenza A virus replication and pneumonia [13]. Herein, emodin is not only an inhibitor of the SNE-encoded 3a protein, but also blocks the interaction between $\mathrm{S}$ protein and angiotensin-converting enzyme 2 (ACE2) [14, 15]. Meanwhile, the latest research findings have indicated that SARS-CoV-2 infection is associated with an affinity of $S$ proteins and ACE2 receptors [16]. However, to date, no exploration of the efficacy of emodin administration against COVID-19, especially its potential targets and mechanisms, has been performed.
Andrew L. Hopkins, a British pharmacologist, first introduced a new discipline based on systems biology [17], which came to be known as network pharmacology. This discipline focuses on the multitargets regulation of signaling pathways. Importantly, it not only promotes the success of clinical trials of novel drugs, but also reduces expenses in drug development [18]. Molecular docking is an approach of drug-design that involves establishing the relationships between molecules and receptors [19], which contributes to the simulation of interactions between compounds and proteins; notably, this helps to enhance the credibility of network pharmacology.

Collectively, to reveal biological targets and signaling pathways connected with the disease, an analysis of network pharmacology and molecular docking technology of emodin against COVID-19 was carried out. In this research (Figure 1), we first identified potential targets of emodin in COVID-19, and then investigated the intersection of the drug targets and genes associated with diseases. Additionally, a proteinprotein interaction (PPI) network was constructed to reveal interactions among proteins. Gene Ontology (GO) and Kyoto Encyclopedia of Genes and Genomes (KEGG) enrichment analysis were performed based on core targets. Eventually, we performed molecular docking to verify the interactions between emodin and its selected targets. Our findings may help to elucidate the pharmacological mechanism of emodin against COVID-19 and increase the possibility of developing novel therapeutics for this condition.

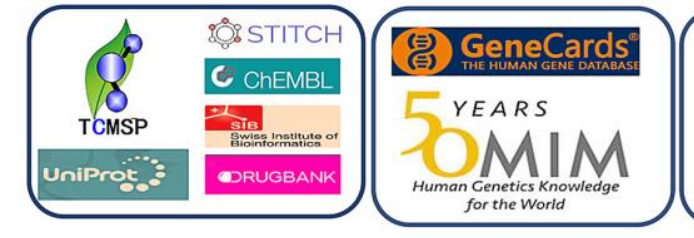

Collection of Emodin Targets

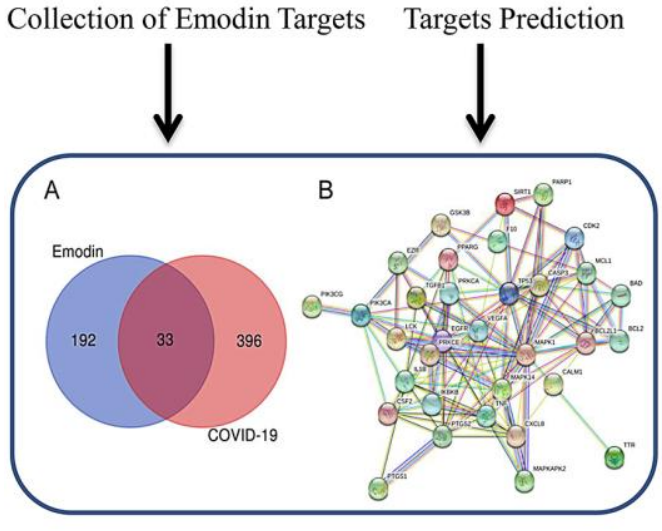

Emodin Targets
COVID-19 Targets
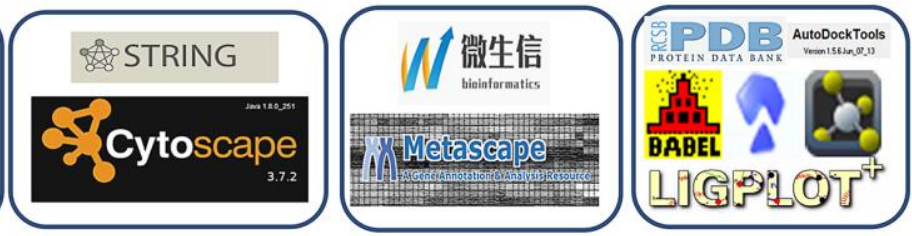

Network Analysis

Pathway Analysis

Molecular Verification
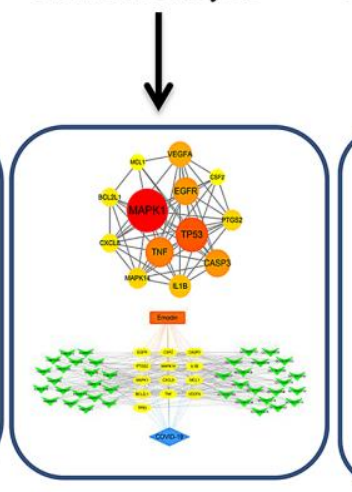

Network Construction

GO Enrichment and KEGG Pathway Analysis

Figure 1. Flow diagram of network pharmacological research on emodin against COVID-19. 


\section{MATERIALS AND METHODS}

\section{Screening of candidate genes associated with emodin}

To obtain potential pharmacological targets associated with emodin, online databases were used. These databases include Traditional Chinese Medicine Systems Pharmacology (TCMSP, https://tcmspw.com/tcmsp.php, version 2.3), Drugbank (https://www.drugbank.ca/, updated September 26, 2020), STITCH (http://stitch.embl.de/, updated September 26, 2020), Swiss Target Prediction (http://www.swisstargetprediction.ch/, updated September 26, 2020), and ChEMBL (https://www.ebi.ac.uk/chembl/, updated September 26, 2020). These genes were input into the Uniprot (https://www.uniprot.org/, updated September 28, 2020) database and set to "Homo sapiens" to ensure accurate target harvesting.

\section{Collection of candidate genes associated with COVID-19}

To collect targets affiliated with COVID-19, the Human Gene Database (GeneCards, https://www.genecards.org/, updated September 25, 2020) and Online Mendelian Inheritance in Man (OMIM, https://www.omim.org/, updated September $25,2020)$ were chosen. The whole primary genes or targets of emodin and COVID-19 were identified and subjected to intersection analysis via an online platform in the form of Venn diagrams (http://bioinformatics.psb.ugent.be/webtools/Venn/) to display the correlation between emodin-activated genes and their targets against COVID-19.

\section{Establishment of PPI network}

The selected therapeutic targets of emodin against COVID-19 were input into the STRING (Version: 11.0, https://string-db.org/) database for PPI network analysis. Furthermore, the Cytoscape V3.7.2 plugin Network Analyzer was applied to analyze the network topology data through a series of parameters, such as degree centrality in the network. As a result, core targets were selected based on degree values. For filtering, the upper limit chosen was the maximum degree-value in the topology data, while the lower limit was the median of freedom as previously reported [20].

\section{Gene Ontology (GO) and Kyoto encyclopedia of genes and genomes (KEGG) pathway enrichment analysis}

The potential core targets of emodin against COVID-19 were imported into the Metascape (http://metascape.org) database [21] by entering the list of target gene names and selecting "Homo sapiens" as the species for GO enrichment and KEGG pathway analysis. Go enrichment analysis generally includes three ontologies: biological process (BP), cellular component (CC), and molecular function (MF). To perform GO enrichment and KEGG pathway analysis, the statistical significance threshold was set at $P<0.01$, and the WeChat online mapping website was used to visualize the analysis results.

\section{Verification of interactions between targets and emodin through molecular docking}

The three-dimensional structure of emodin was downloaded from the PubChem database [22]. Subsequently, the MOL2 file was transformed into a PDB format using Open Babel 2.4.1 software. Then, we converted the PDB file into a pdbqt format using the AutoDock Tools v.1.5.6 software. The receptor proteins, including 3CL (PDB ID: 6LU7) and ACE2 (PDB ID:2AJF), were obtained from RCSB Protein Data Bank (PDB, http://www.rcsb.org/) database [23]. During the docking process, the proteins were imported into PyMOL 2.3.2 software to remove water molecules, and the Autodock Tools v.1.5.6 software was used to conduct hydrogenation and to calculate Gasteiger charges; these results were subsequently saved as the pdbqt format. The parameters of the receptor protein docking sites were set to cover those active pocket sites in which small molecular ligands complex might bind. In addition, the ligand compound (emodin) was set to flexibility and the receptor to rigidity. Furthermore, AutoDock Vina v.1.1.2 [24] was used to dock the compound and core targets or proteins, including the proteins of 3CL and ACE2 in SARS-CoV-2. The visualization of the docking results with the lowest binding energy was presented using PyMOL 2.3.2 software and LigPlot $+v 2.2$.

\section{RESULTS}

\section{Screening of the genes of emodin and COVID-19}

To determine the genes associated with COVID-19 and the therapeutic targets of emodin, a series of bioinformatics analyses were carried out. We screened and identified a total of 429 COVID-19-associated genes from the Genecards and OMIM databases. Meanwhile, we also identified 225 pharmacodynamic targets of emodin from TCMSP, Drugbank, STITCH, Swiss Target Prediction, and ChEMBL databases. Subsequently, the aforementioned 429 targets were combined with these 225 targets of emodin, and a total of 33 potential therapeutic targets of emodin against COVID-19 were revealed (Figure 2A). Then the 
common genes were established in a PPI network diagram (Figure 2B) and we analyzed the PPI relationships using the STRING database.

The data of the above PPI network were input into Cytoscape V3.7.2 software to acquire the information of the topology parameters. The results showed that the median degree of freedom was 8 , and the maximum degree of freedom was 21. As shown in Figure 3, the top 13 targets of emodin against COVID-19, including mitogen-activated protein kinase 1 (MAPK1), tumor protein (TP53), tumor necrosis factor (TNF), caspase-3 (CASP3), epidermal growth factor receptor (EGFR), vascular endothelial growth factor A (VEGFA),
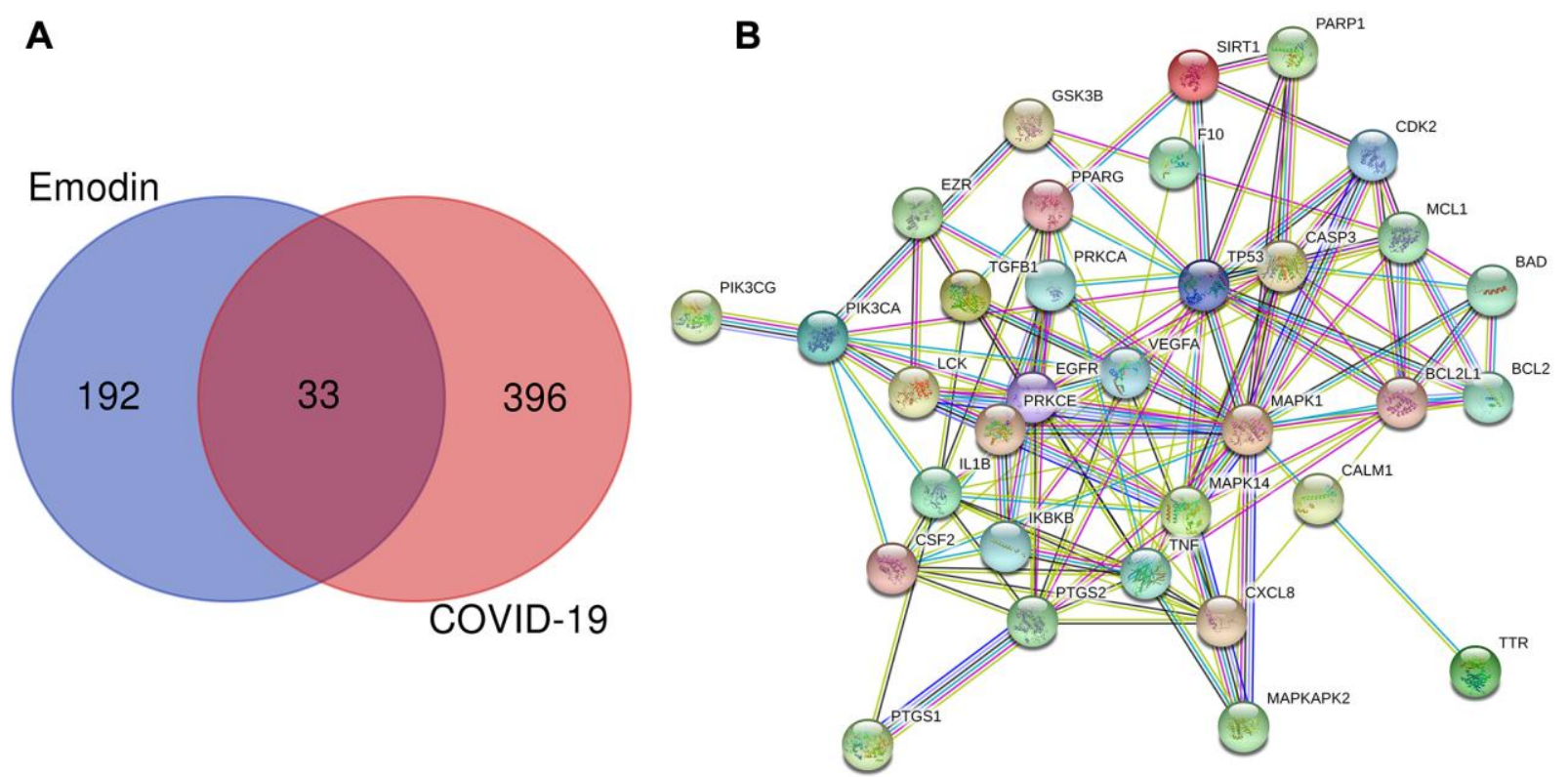

Figure 2. (A) Venn diagram was applied to present all candidate targets of emodin and COVID-19. (B) PPI network visualization of the potential therapeutic targets of emodin against COVID-19. The network nodes depict target proteins, and the edges represent proteinprotein relationships.

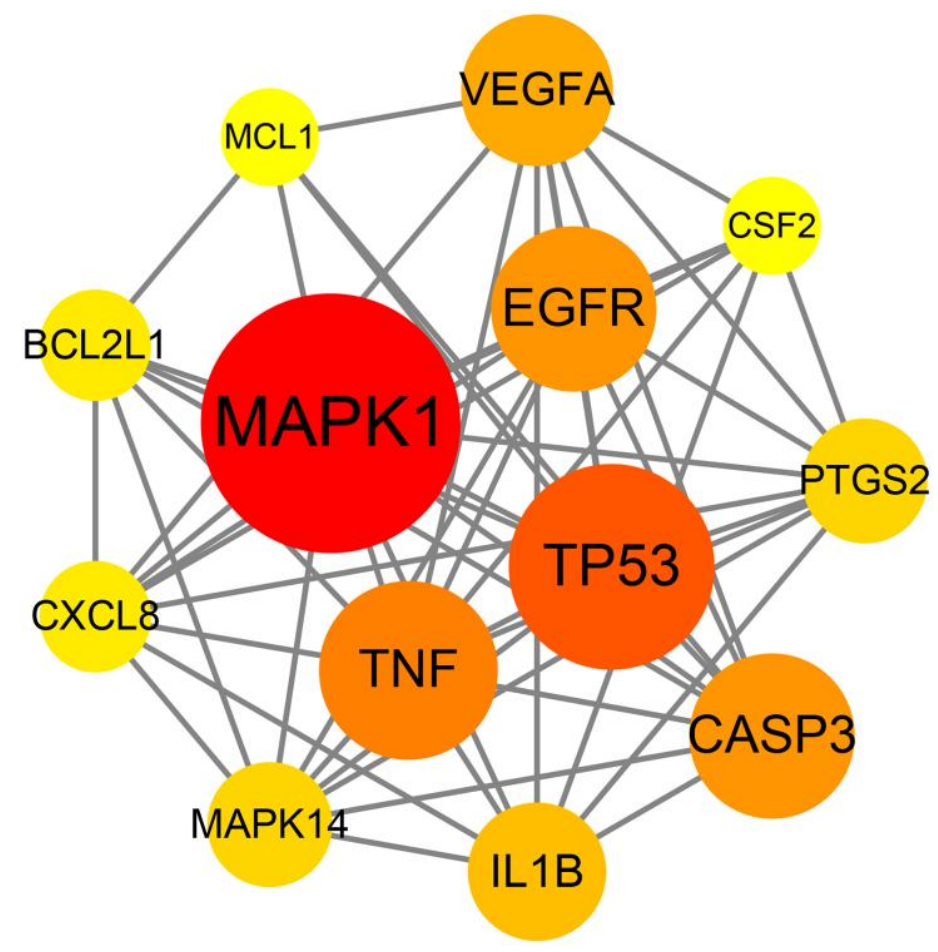

Figure 3. Network diagram of the core targets of emodin against COVID-19. 
interleukin 1B (IL1B), mitogen-activated protein kinase 14 (MAPK14), prostaglandin-endoperoxide synthase 2 (PTGS2), B-cell lymphoma-2-like protein 1 (BCL2L1), interleukin-8 (CXCL8), myeloid cell leukemia 1 (MCL1), and colony stimulating factor (CSF2), had a higher number of connections than other genes and were identified as core targets.

\section{Analysis of biological features of emodin against COVID-19}

To gain insight into the potential biological features and signaling pathways of emodin against COVID-19, we performed functional annotation of the core targets based on GO enrichment and KEGG pathway analysis. Total 543 GO terms were selected, of which BP accounted for 514, CC for 11, and MF for 18. As shown in Figure 4, among them, most GO terms associated with the BP category were correlated with cytokine- mediated signaling pathway, signal transduction in absence of ligand, extrinsic apoptotic signaling pathway in the absence of ligand, response to lipopolysaccharide, response to molecule of bacterial origin, developmental process involved in reproduction, reproductive structure development, etc. The $\mathrm{CC}$ enrichment analysis mainly included the following concepts: membrane raft, membrane microdomain, membrane region, vesicle lumen, nuclear membrane, secretory granule lumen, cytoplasmic vesicle lumen, nuclear envelope, adherens junction, anchoring junction, etc. Simultaneously, MF annotation mainly presented cytokine receptor binding, cytokine activity, phosphatase binding, receptor-ligand activity, receptor regulator activity, protein kinase binding, kinase binding, protease binding, growth factor receptor binding, protein phosphatase binding, etc. Interestingly, as shown in Figure 5, the core targets of emodin against COVID-19 were directly related with the top 10 terms of the GO BP category, while the top

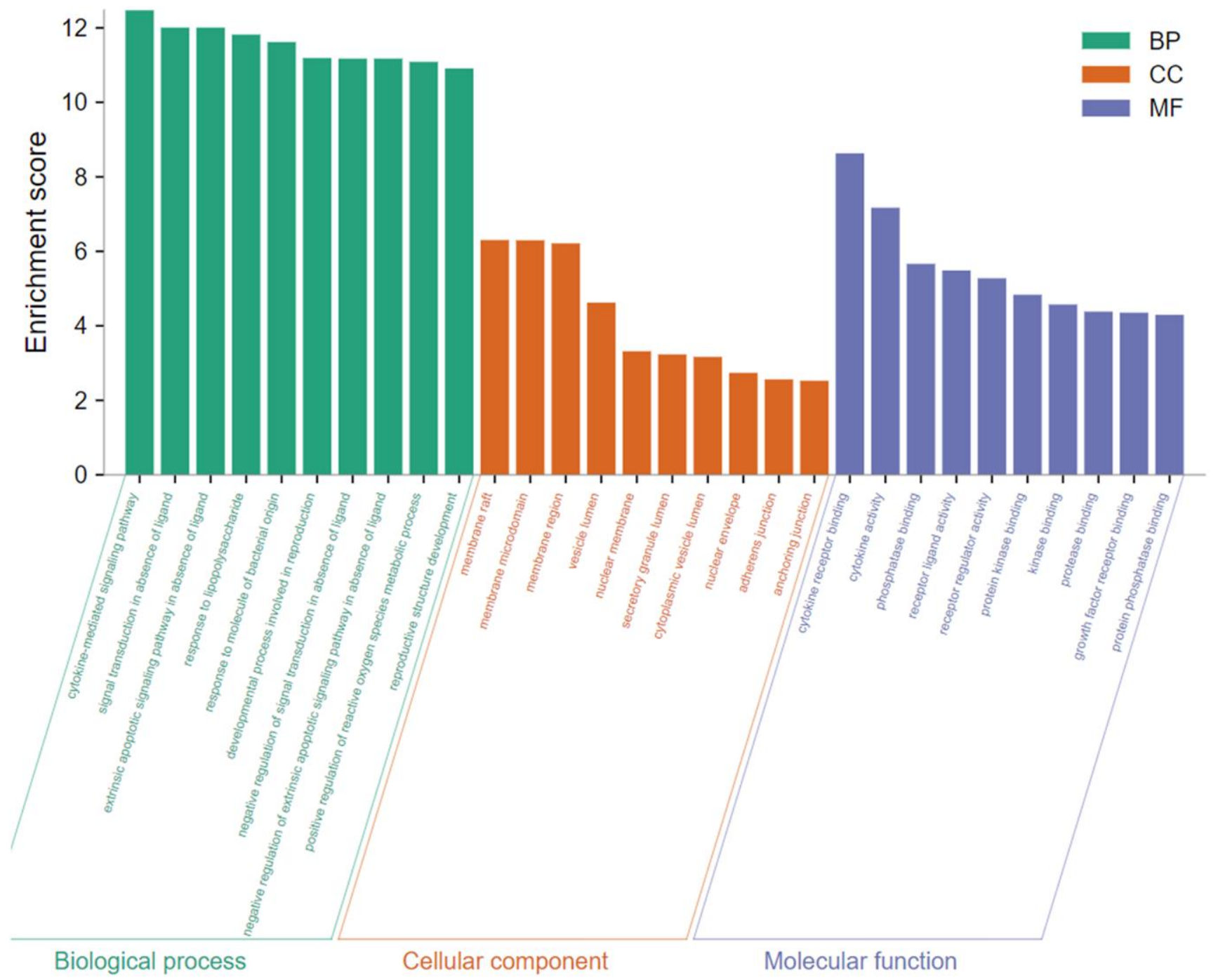

Figure 4. The top 10 of biological process, cellular component, and molecular function from GO terms analysis were illustrated by bar diagrams with enrichment scores (the x-axis represents top $10 \mathrm{GO}$ terms, and the $y$-axis indicates the $p$-value). 
10 terms of the BP category had many targets in common, suggesting that the core targets of emodin may be involved in various regulatory processes of $\mathrm{BP}$ to resist COVID-19 effects.

\section{Analysis of emodin-mediated signaling pathway and network against COVID-19}

To further understand the potential mechanisms of emodin against COVID-19, KEGG pathway enrichment was carried out. From data analysis, a total of 72 pathways were enriched based on the adjusted $p$-value parameter $(P<0.01)$. The KEGG pathway results demonstrated that the main pathways included IL-17 signaling pathway, AGE-RAGE signaling pathway in diabetic complications, TNF signaling pathway, pertussis, proteoglycans in cancer, and other contagious pulmonary diseases. Apart from the above, we also found that emodin could mediate many signaling pathways, including MAPK signaling pathway, NODlike receptor signaling pathway, NF-kappa B signaling pathway, as well as many pathways related to

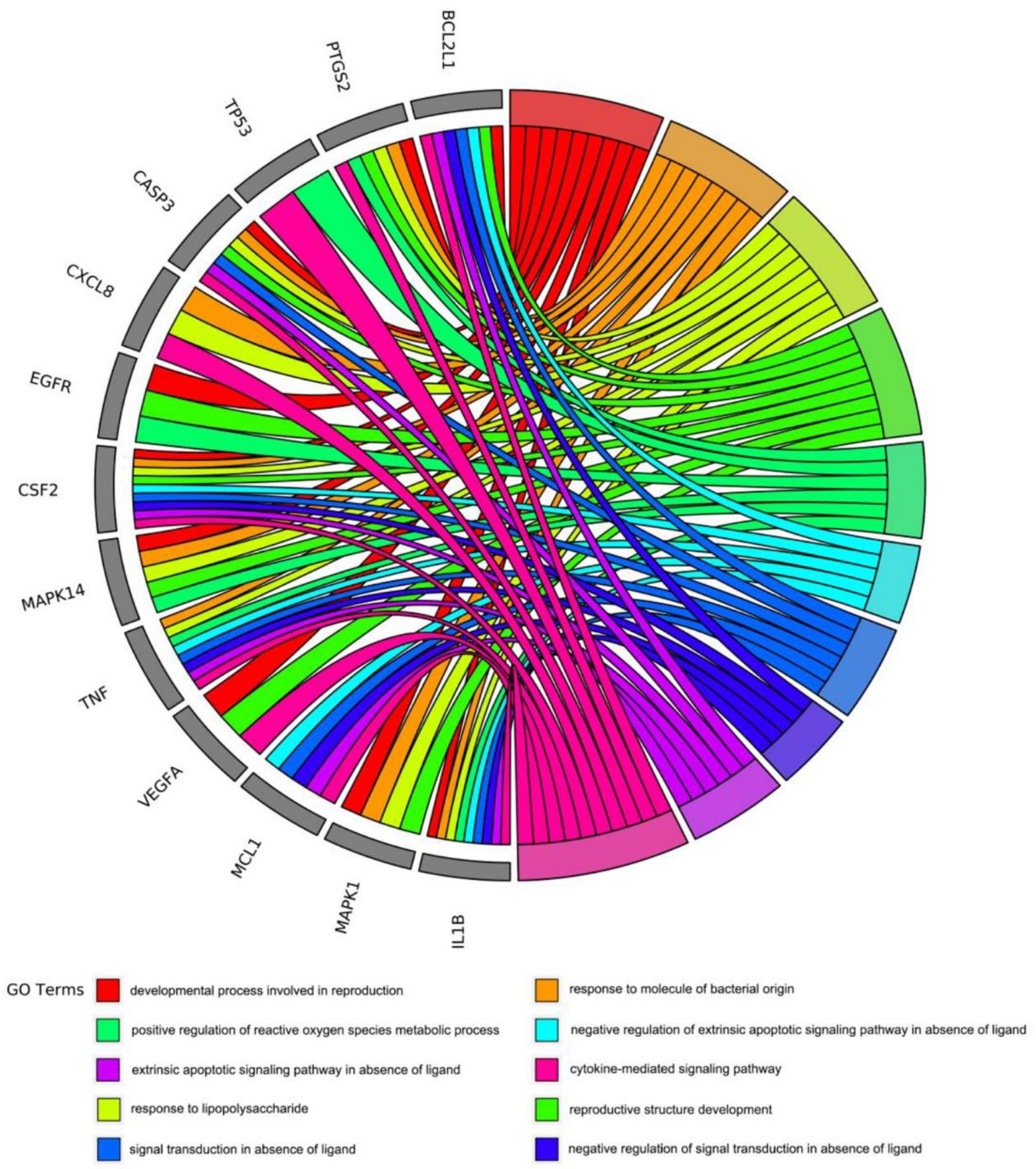

Figure 5. The core targets of emodin-treated COVID-19 were linked to the top 10 most enriched GO BP terms in GO chord plot. 
inflammatory or immune responses (Figure 6). As shown in Figure 7, thirteen core targets of emodin against COVID-19 were directly associated with the top 11 pathways, indicating that the core targets of emodin could be treatments of COVID-19 by acting on multiple pathways. We analyzed the potential mechanisms in the treatment of COVID-19. Based on network pharmacology, we established the interaction network diagram of "emodin-target-GO-KEGG-COVID-19" by the application of Cytoscape V3.7.2 software (Figure 8).

Subsequently, we created network diagrams which highlighted core targets of KEGG-enriched pathways using the 'pathview' package in $R$ for emodin against COVID-19 (Supplementary Figure 1-5).

\section{Results of molecular docking}

Molecular docking analysis predicted the binding capability between emodin and the 15 tested target proteins (Table 1). In this analysis, the value of the docking score indicates the binding activity between the compound and the protein. The lower the docking score, the more stable the binding conformation, and the greater the possibility of molecular interaction. Based on a binding energy of less than $-7.0 \mathrm{kcal} / \mathrm{mol}$, ACE2, 3CL, PTGS2, CSF2, EGFR, and TP53 of the 15 tested target proteins were selected. As indicated in Figure 9, in terms of interaction points, emodin mainly interacted with amino acid residues Asp206, Glu208 and Gly98 in ACE2. Meanwhile, emodin mainly interacted with 3CL via Gln19, Gln69, Met17 and Lys97 (amino acid residues) and could bind to PTGS2 amino acid residues Gln461, Glu465 and His39. Also, emodin mainly interacted with CSF2 bridged with amino acid residues (Trp13, Glu14, His15, and Gln86). In the EGFR protein, emodin formed hydrogen bonds with amino acid residues Val726, Gly724, Phe723, Asp837, Arg841, Asn842 and Gly719. Finally, the hydrogen bonding between emodin and the TP53 protein acted on the amino acid residues Arg202, Thr155, Pro151 and Pro152.

\section{Top 20 of Pathway Ehrichment}

IL-17 signaling pathwayAGE-RAGE signaling pathway in diabetic complications TNF signaling pathway -

Pertussis -

Proteoglycans in cancerPathways in cancer -

Bladder cancer MAPK signaling pathway Hepatitis C -

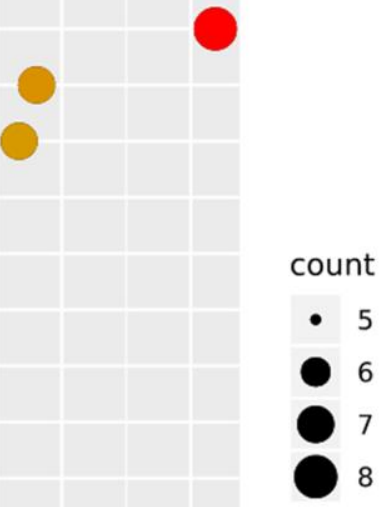

Amyotrophic lateral sclerosis (ALS) -

Apoptosis -

MicroRNAs in cancer -

Pancreatic cancer -

NOD-like receptor signaling pathway -

Leishmaniasis -

Salmonella infection -

Rheumatoid arthritis -

NF-kappa B signaling pathway - ○

Amoebiasis - 0

Chagas disease (American trypanosomiasis) - ○

Figure 6. The top 20 signaling pathways from KEGG enrichment analysis were showed by the bubble diagram with count algorithms and $\boldsymbol{p}$-values. Each node signals one KEGG pathway, and its size represents the gene number. The color indicates the $p$-value. 


\section{DISCUSSION}

With the outbreak of COVID-19, more attention to the transmission and effects of SARS-CoV-2 has been paid worldwide. Confirmed cases of COVID-19 have sharply increased in number over time despite the novelty of the causative virus. Prior works by researchers have reported that although emodin possesses potential efficacy against the SARS virus [15], the mechanism of emodin against COVID-19 is not clear. Herein, we revealed a pharmacological mechanism of emodin against COVID-9 using integrative network pharmacology and other bioinformatics technologies. Our results revealed that the top 20 pathways were mainly involved in viral infections, inflammation, immune responses, cancer, and other contagious pulmonary diseases. Thus, we could speculate that emodin may contribute to the treatment of COVID-19 via regulating inflammationrelated pathways, immune regulation-related pathways, and viral infection-related pathways. Moreover, in the PPI network, the top 13 targets, including BCL2L1, PTGS2, TP53, CASP3, CXCL8, EGFR, CSF2, MAPK14, TNF, VEGFA, MCL1, MAPK1, and IL1B,

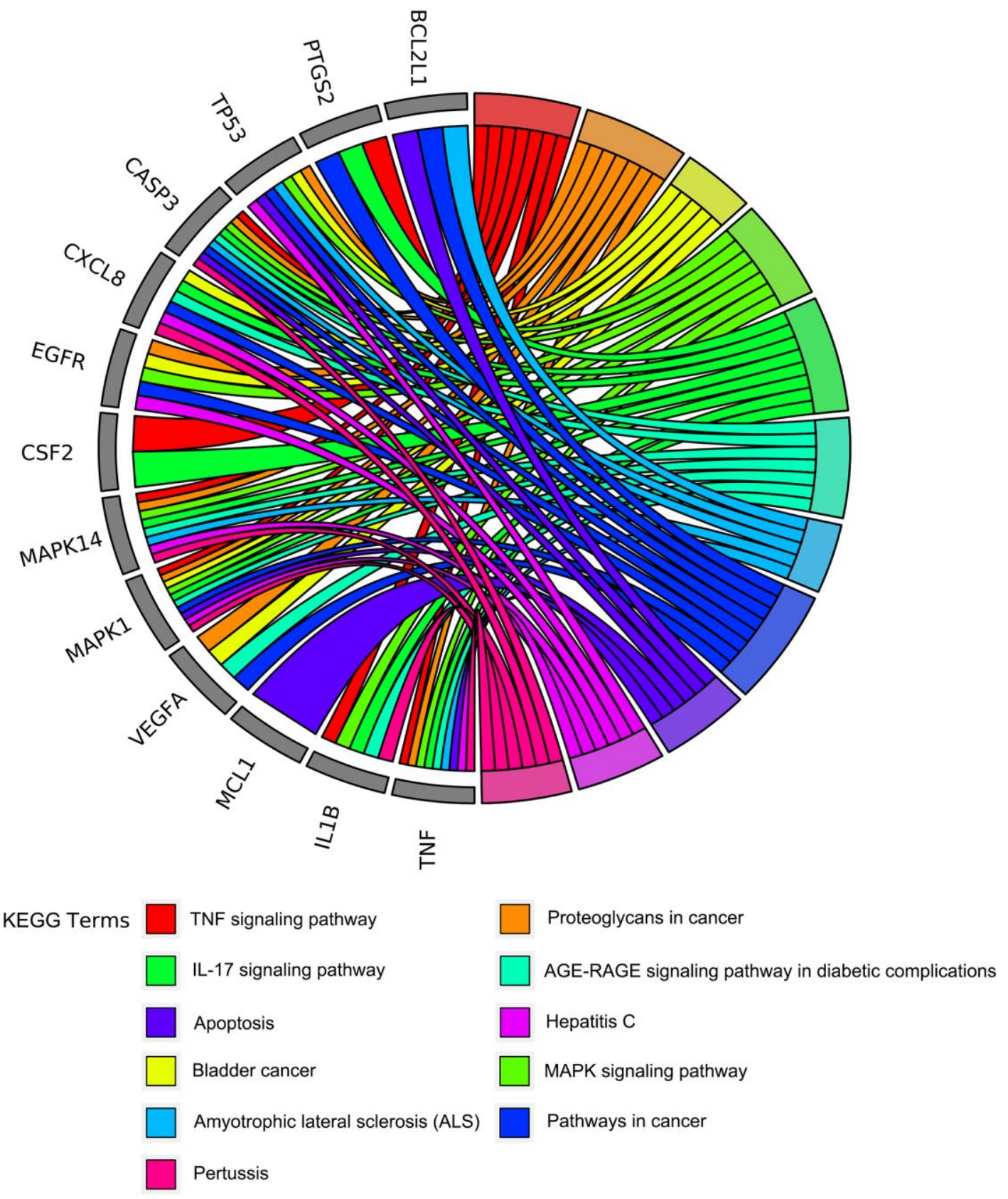

Figure 7. The circo-diagrams of the top 11 ranked overrepresented KEGG pathways. Different colors on the right side of the graph represent different signaling pathways, and the left side is the top 13 core targets of emodin against COVID-19 with relevance. 
Table 1. Binding energy for core targets.

\begin{tabular}{lccc}
\hline Targets & Binding energy (kcal/mol) & Targets & Binding energy $(\mathbf{k c a l} / \mathbf{m o l})$ \\
\hline MAPK1 & -6.87 & PTGS2 & -8.51 \\
TP53 & -7.03 & BCL2L1 & -5.77 \\
TNF & -6.46 & CXCL8 & -5.80 \\
CASP3 & -6.96 & MCL1 & -5.99 \\
EGFR & -7.65 & CSF2 & -7.97 \\
VEGFA & -6.10 & 3 CL & -7.04 \\
IL1B & -6.97 & ACE2 & -7.26 \\
MAPK14 & -6.69 & & \\
\hline
\end{tabular}

were selected as core targets that may play vital roles in emodin against COVID-19. These core targets were enriched in the different signaling pathways. We obtained some pathways related to immune responses, bacterial structure and reproduction, inflammatory response diabetic complications, and cancers based on GO enrichment and KEGG pathway analysis.

Cytokine-mediated signaling pathway can participate in severe inflammatory responses, which may lead to pneumonia in people with COVID-19 [25]. Due to the release of tissue factor in response to cytokines triggered by endothelial cells, it may further progress to the formation of coagulation in COVID-19 patients [26]. In addition, lipopolysaccharides cause inflammatory effects and are also pro-inflammatory compounds of bacterial origin [27]. Importantly, the function of lipopolysaccharide or molecule of bacterial origin provides viruses with thermal stabilization [28]. The reproduction number is an important parameter by which transmissibility can be measured during epidemics [29]. A prior study by Khajanchi et al. [30] reported a higher value, suggesting the possibility of an outbreak of COVID-19 phenomena in India. Administration of emodin may facilitate the host to induce anti-inflammatory response to achieve antiCOVID-19 effects.

Our KEGG pathway analysis uncovered that these pathways were correlated with immune response, inflammatory response, and the recognition of pathogen-associated molecular modes. KEGG pathway analysis exhibited that some related signaling pathways were affected by emodin in the treatment of COVID-19, including IL-17 signaling pathway, MAPK signaling pathway, NF-kappa B signaling pathway, NOD-like receptor signaling pathway, and TNF signaling pathway, as presented by KEGG pathway analysis (Supplementary Figure 1-5). The IL-17 signaling pathway is critical for clearing extracellular pathogens and is associated with acute respiratory distress syndrome [31]. One study demonstrated that there is the

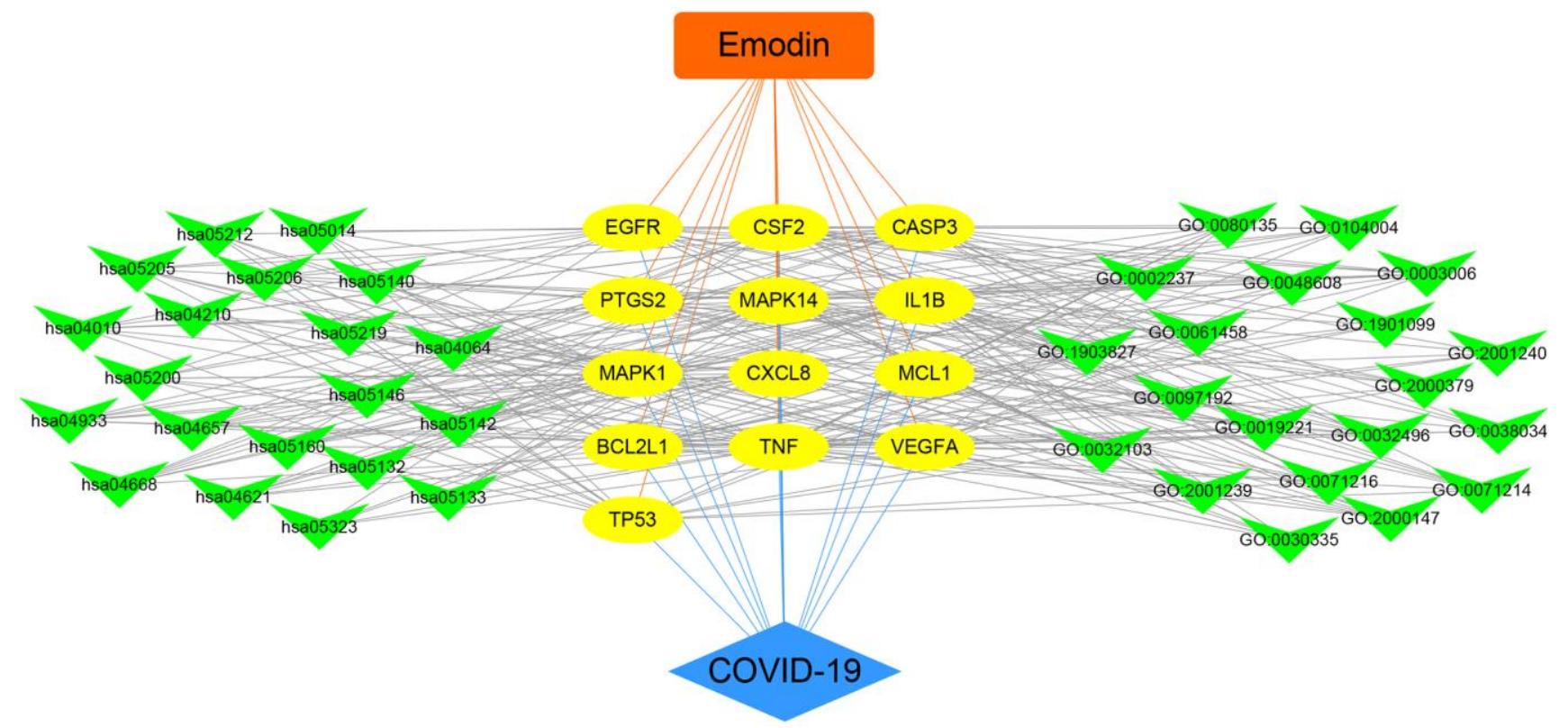

Figure 8. Integrated visualization of interaction network of target-disease-function-pathway in emodin-treated COVID-19. 
elevation of IL-17 in bronchoalveolar lavage fluid in patients with acute respiratory distress syndrome [32]. Another study showed that the limitation of IL-17 signaling can inhibit the lung inflammation in mice [33], indicating a potential mechanism in the treatment of COVID-19 via the reduction of IL-17 levels. Meanwhile, the activation of IL-17 can enhance the expression of cytokines [34], which can be found in patients with COVID-19. The downstream signaling of IL-17 is linked to NF-kappa B signaling and MAPK signaling. IL-17 also boosts the generation of chemokines and develops the recruitment of immune cells [35, 36]. Other researchers have found that the expression levels of several inflammatory factors are increased in patients with COVID-19 by way of a quantitative proteomics approach combined with bioinformatics analyses, which is possibly caused by the activation of NF-kappa B signaling [37]. Similarly,
MAPK signaling inactivation contributes to a reduced the chance of COVID-19 [38, 39]. Prior studies have suggested that elevated pro-inflammatory IL-17 is associated with elevated NOD-like receptor [40, 41]. NOD-like receptor, one of the inflammatory components, can increase the expression of interleukin$1 \beta$ [35]. Interleukin can regulate the immune response and is involved in the inflammatory response. Apart from the above, elevated NOD-like receptor genes lead to the degradation of the viral genome and inhibition of the virus [42].

In addition, patients with COVID-19 also have many complications, such as diabetes and cancer. ACE2, the SARS-CoV-2 receptor, can increase the risk of COVID19 in patients with diabetes in clinic [43]. Also, there is a clue that ACE2-positive expression in diabetic disease was observed in SARS-CoV-2 infected cells [44]. These
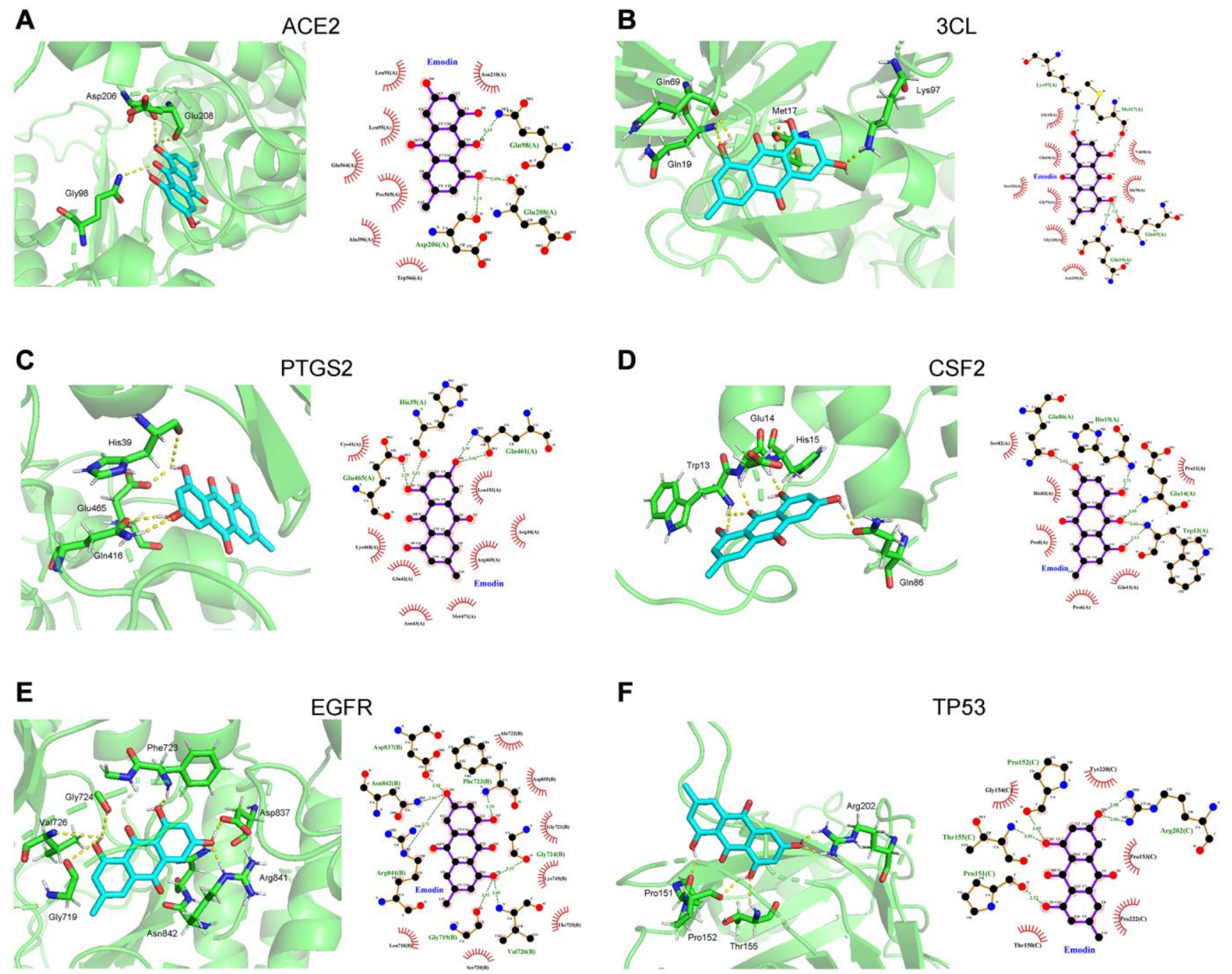

Figure 9. Molecular docking diagram showed that the binding capability of emodin against COVID-19 was presented as 3D diagrams and 2D diagrams, respectively. (A) Angiotensin converting enzyme 2 (ACE2), (B) 3CL. (C) Prostaglandin endoperoxide synthase 2 (PTGS2). (D) Colony stimulating factor 2 (CSF). (E) Epidermal growth factor receptor (EGFR). (F) Tumor protein 53 (TP53). 
findings support that emodin administration may improve the symptoms of COVID-19 via regulating targets related to diabetes.

Molecular docking presents a tool that contributes to the discovery or design of drugs. According to the literature, AEC2 and 3CL proteins serve as direct targets for blocking COVID-19 [1]. Meanwhile, the proteins of CFS2 and PTGS2 are associated with inflammation [45], and EGFR and TP53 regulate the immune response [46]. Based on the aforementioned molecular docking results, the scores of emodin with the key targets were all less than $-5.0 \mathrm{kcal} / \mathrm{mol}$, of which 6 targets had a score less than $-7.0 \mathrm{kcal} / \mathrm{mol}$. Their interaction sites or points were uncovered, indicating that emodin had good binding activities with ACE2, 3CL, PTGS2, CSF2, EGFR, and TP53, respectively. Therefore, based on our data, emodin shows great potential for treating COVID-19. Although its efficacy still needs to be verified by further experimental work.

\section{CONCLUSIONS}

Briefly, network pharmacology revealed all core targets, biological function, and signaling pathways of emodin against COVID-19. The present results showed that emodin could contribute to treating COVID-19 via the regulation of inflammation-related pathways, immune regulation-related pathways, and viral infection-related pathways. Furthermore, these molecular docking results indicated the potential benefits for supporting the possibility of emodintreated COVID-19 in clinic. According to our data, emodin may serve as a promising candidate drug for the treatment for COVID-19.

\section{AUTHOR CONTRIBUTIONS}

JY and HW conceived and designed this study. HD, $\mathrm{JZ}$ and JC performed the study and analyzed the data. HZ supervised the study. HD and JZ wrote and revised the paper. All authors were responsible for reviewing data. All authors read and approved the final manuscript.

\section{CONFLICTS OF INTEREST}

The authors declare no conflicts of interest related to this study.

\section{FUNDING}

This study was financially supported by the State Key Program of National Natural Science Foundation of
China (No. 81930111), Outstanding Talents of Ten Thousand Talents Program of Zhejiang Province (No. 2019R51002), and the Key Research and Development Program in Zhejiang Province (No. 2020C03052).

\section{REFERENCES}

1. Huang YF, Bai C, He F, Xie Y, Zhou H. Review on the potential action mechanisms of Chinese medicines in treating Coronavirus Disease 2019 (COVID-19). Pharmacol Res. 2020; 158:104939. https://doi.org/10.1016/i.phrs.2020.104939 PMID:32445956

2. Luo L, Liu D, Liao X, Wu X, Jing $Q$, Zheng J, Liu F, Yang S, Bi H, Li Z, Liu J, Song W, Zhu W, et al. Contact Settings and Risk for Transmission in 3410 Close Contacts of Patients With COVID-19 in Guangzhou, China: A Prospective Cohort Study. Ann Intern Med. 2020; 173:879-87. https://doi.org/10.7326/M20-2671 PMID: $\underline{32790510}$

3. Dai WC, Zhang HW, Yu J, Xu HJ, Chen H, Luo SP, Zhang $\mathrm{H}$, Liang $\mathrm{LH}, \mathrm{Wu} \mathrm{XL}$, Lei $\mathrm{Y}$, Lin $\mathrm{F}$. CT Imaging and Differential Diagnosis of COVID-19. Can Assoc Radiol J. 2020; 71:195-200. https://doi.org/10.1177/0846537120913033 PMID: $\underline{32129670}$

4. Yang R, Liu $H$, Bai C, Wang $Y$, Zhang $X$, Guo R, Wu $S$, Wang J, Leung $E$, Chang $H$, Li P, Liu T, Wang $Y$. Chemical composition and pharmacological mechanism of Qingfei Paidu Decoction and Ma Xing Shi Gan Decoction against Coronavirus Disease 2019 (COVID-19): In silico and experimental study. Pharmacol Res. 2020; 157:104820.

https://doi.org/10.1016/j.phrs.2020.104820 PMID:32360484

5. Lymperopoulos IN. \#stayhome to contain Covid-19: Neuro-SIR - Neurodynamical epidemic modeling of infection patterns in social networks. Expert Syst Appl. 2021; 165:113970. https://doi.org/10.1016/j.eswa.2020.113970 PMID:32908331

6. Chandra TB, Verma K, Singh BK, Jain D, Netam SS. Coronavirus disease (COVID-19) detection in Chest XRay images using majority voting based classifier ensemble. Expert Syst Appl. 2021; 165:113909. https://doi.org/10.1016/j.eswa.2020.113909 PMID: $\underline{32868966}$

7. Zhao Z, Li Y, Zhou L, Zhou X, Xie B, Zhang W, Sun J. Prevention and treatment of COVID-19 using Traditional Chinese Medicine: A review. 
Phytomedicine. 2021; 85:153308.

https://doi.org/10.1016/i.phymed.2020.153308

PMID:32843234

8. Wu CC, Chen MS, Cheng YJ, Ko YC, Lin SF, Chiu IM, Chen JY. Emodin Inhibits EBV Reactivation and Represses NPC Tumorigenesis. Cancers (Basel). 2019; 11:1795.

https://doi.org/10.3390/cancers11111795 PMID:31731581

9. Fang L, Zhao F, Iwanowycz S, Wang J, Yin S, Wang Y, Fan D. Anticancer activity of emodin is associated with downregulation of CD155. Int Immunopharmacol. 2019; 75:105763.

https://doi.org/10.1016/j.intimp.2019.105763 PMID:31325728

10. Jung J, Kim NK, Park S, Shin HJ, Hwang SG, Kim K. Inhibitory effect of Phyllanthus urinaria L. extract on the replication of lamivudine-resistant hepatitis B virus in vitro. BMC Complement Altern Med. 2015; 15:255.

https://doi.org/10.1186/s12906-015-0792-3 PMID:26220282

11. Ma C, Wen B, Zhang Q, Shao PP, Gu W, Qu K, Shi Y, Wang B. Emodin induces apoptosis and autophagy of fibroblasts obtained from patient with ankylosing spondylitis. Drug Des Devel Ther. 2019; 13:601-09. https://doi.org/10.2147/DDDT.S182087 PMID:30809091

12. Irais CM, María-de-la-Luz SG, Dealmy DG, Agustina RM, Nidia CH, Mario-Alberto RG, Luis-Benjamín SG, María-Del-Carmen VM, David PE. Plant Phenolics as Pathogen-Carrier Immunogenicity Modulator Haptens. Curr Pharm Biotechnol. 2020; 21:897-905. https://doi.org/10.2174/1389201021666200121130313 PMID:31965941

13. Dai JP, Wang QW, Su Y, Gu LM, Zhao Y, Chen XX, Chen C, Li WZ, Wang GF, Li KS. Emodin Inhibition of Influenza A Virus Replication and Influenza Viral Pneumonia via the Nrf2, TLR4, p38/JNK and NFkappaB Pathways. Molecules. 2017; 22:1754. https://doi.org/10.3390/molecules22101754 PMID:29057806

14. Schwarz S, Wang K, Yu W, Sun B, Schwarz W. Emodin inhibits current through SARS-associated coronavirus 3a protein. Antiviral Res. 2011; 90:64-69. https://doi.org/10.1016/i.antiviral.2011.02.008 PMID:21356245

15. Ho TY, Wu SL, Chen JC, Li CC, Hsiang CY. Emodin blocks the SARS coronavirus spike protein and angiotensin-converting enzyme 2 interaction. Antiviral Res. 2007; 74:92-101. https://doi.org/10.1016/j.antiviral.2006.04.014
PMID:16730806

16. Dong J, Huang B, Jia Z, Wang B, Gallolu Kankanamalage S, Titong A, Liu Y. Development of multi-specific humanized llama antibodies blocking SARS-CoV-2/ACE2 interaction with high affinity and avidity. Emerg Microbes Infect. 2020; 9:1034-36. https://doi.org/10.1080/22221751.2020.1768806 PMID:32403995

17. Wu W, Zhang Z, Li F, Deng Y, Lei $M$, Long $H$, Hou J, Wu W. A Network-Based Approach to Explore the Mechanisms of Uncaria Alkaloids in Treating Hypertension and Alleviating Alzheimer's Disease. Int J Mol Sci. 2020; 21:1766. https://doi.org/10.3390/ijms21051766 PMID:32143538

18. Fang T, Liu L, Liu W. Network pharmacology-based strategy for predicting therapy targets of Tripterygium wilfordii on acute myeloid leukemia. Medicine (Baltimore). 2020; 99:e23546. https://doi.org/10.1097/MD.0000000000023546 PMID: $\underline{3327305}$

19. Hannan MA, Dash $R$, Haque MN, Mohibbullah $M$, Sohag AAM, Rahman MA, Uddin MJ, Alam M, Moon IS. Neuroprotective Potentials of Marine Algae and Their Bioactive Metabolites: Pharmacological Insights and Therapeutic Advances. Mar Drugs. 2020; 18:347. https://doi.org/10.3390/md18070347 PMID:32630301

20. Li R, Song Y, Ji Z, Li L, Zhou L. Pharmacological biotargets and the molecular mechanisms of oxyresveratrol treating colorectal cancer: Network and experimental analyses. Biofactors. 2020; 46:15867.

https://doi.org/10.1002/biof.1583 PMID:31647596

21. Zhang S, Song G, Yuan J, Qiao S, Xu S, Si Z, Yang Y, Xu $X$, Wang A. Circular RNA circ_0003204 inhibits proliferation, migration and tube formation of endothelial cell in atherosclerosis via miR-3703p/TGFßR2/phosph-SMAD3 axis. J Biomed Sci. 2020; 27:11.

https://doi.org/10.1186/s12929-019-0595-9 PMID:31900142

22. Kim S, Chen J, Cheng T, Gindulyte A, He J, He S, Li Q, Shoemaker BA, Thiessen PA, Yu B, Zaslavsky L, Zhang J, Bolton EE. PubChem 2019 update: improved access to chemical data. Nucleic Acids Res. 2019; 47:D1102-09. https://doi.org/10.1093/nar/gky1033 PMID: $\underline{30371825}$

23. Unni S, Deshmukh P, Krishnappa G, Kommu $P$, Padmanabhan B. Structural insights into the multiple binding modes of Dimethyl Fumarate (DMF) and its 
analogs to the Kelch domain of Keap1. FEBS J. 2021; 288:1599-613.

https://doi.org/10.1111/febs.15485

PMID:32672401

24. Trott O, Olson AJ. AutoDock Vina: improving the speed and accuracy of docking with a new scoring function, efficient optimization, and multithreading. J Comput Chem. 2010; 31:455-61. https://doi.org/10.1002/jcc.21334 PMID:19499576

25. Thomas DL. Caring for patients in a new pandemic: the necessity and challenges of observational research. J Clin Invest. 2020; 130:6225-27. https://doi.org/10.1172/JCl143292 PMID:32902413

26. FitzGerald ES, Jamieson AM. Unique transcriptional changes in coagulation cascade genes in SARS-CoV-2infected lung epithelial cells: A potential factor in COVID-19 coagulopathies. bioRxiv. 2020. [Epub ahead of print].

https://doi.org/10.1101/2020.07.06.182972 PMID:32676594

27. Bindels LB, Beck R, Schakman O, Martin JC, De Backer F, Sohet FM, Dewulf EM, Pachikian BD, Neyrinck AM, Thissen JP, Verrax J, Calderon PB, Pot B, et al. Restoring specific lactobacilli levels decreases inflammation and muscle atrophy markers in an acute leukemia mouse model. PLoS One. 2012; 7:e37971. https://doi.org/10.1371/journal.pone.0037971 PMID:22761662

28. Waldman P, Meseguer A, Lucas F, Moulin L, Wurtzer S. Interaction of Human Enteric Viruses with Microbial Compounds: Implication for Virus Persistence and Disinfection Treatments. Environ Sci Technol. 2017; 51:13633-40. https://doi.org/10.1021/acs.est.7b03875 PMID:29116763

29. Cori A, Ferguson NM, Fraser C, Cauchemez S. A new framework and software to estimate time-varying reproduction numbers during epidemics. Am J Epidemiol. 2013; 178:1505-12.

https://doi.org/10.1093/aje/kwt133 PMID:24043437

30. Khajanchi S, Sarkar K. Forecasting the daily and cumulative number of cases for the COVID-19 pandemic in India. Chaos. 2020; 30:071101.

https://doi.org/10.1063/5.0016240 PMID: $\underline{32752627}$

31. Orlov M, Wander PL, Morrell ED, Mikacenic C, Wurfel MM. A Case for Targeting Th17 Cells and IL-17A in SARS-CoV-2 Infections. J Immunol. 2020; 205:892-98. https://doi.org/10.4049/jimmunol.2000554 PMID: $\underline{32651218}$

32. Li JT, Melton AC, Su G, Hamm DE, LaFemina $M$, Howard J, Fang X, Bhat S, Huynh KM, O'Kane CM, Ingram RJ, Muir RR, McAuley DF, et al. Unexpected Role for Adaptive $\alpha \beta T h 17$ Cells in Acute Respiratory Distress Syndrome. J Immunol. 2015; 195:87-95. https://doi.org/10.4049/jimmunol.1500054 PMID:26002979

33. Sodhi CP, Nguyen J, Yamaguchi $Y$, Werts $A D$, Lu $P$, Ladd MR, Fulton WB, Kovler ML, Wang S, Prindle T Jr, Zhang $Y$, Lazartigues ED, Holtzman MJ, et al. A Dynamic Variation of Pulmonary ACE2 Is Required to Modulate Neutrophilic Inflammation in Response to Pseudomonas aeruginosa Lung Infection in Mice. J Immunol. 2019; 203:3000-12.

https://doi.org/10.4049/jimmunol.1900579 PMID: $\underline{31645418}$

34. Jiang $\mathrm{Y}$, Tian $\mathrm{M}$, Lin $\mathrm{W}$, Wang $\mathrm{X}$, Wang $\mathrm{X}$. Protein Kinase Serine/Threonine Kinase 24 Positively Regulates Interleukin 17-Induced Inflammation by Promoting IKK Complex Activation. Front Immunol. 2018; 9:921.

https://doi.org/10.3389/fimmu.2018.00921 PMID:29760709

35. Wang L, Lu R, Wang $Y$, Wang $X$, Hao D, Wen X, Li Y, Zeng $M$, Jiang $X$. Identification of Long Noncoding RNA Associated ceRNA Networks in Rosacea. Biomed Res Int. 2020; 2020:9705950. https://doi.org/10.1155/2020/9705950 PMID: 32185228

36. Lucena AMM, Souza CRM, Jales JT, Guedes PMM, de Miranda GEC, de Moura AMA, Araújo-Júnior JX, Nascimento GJ, Scortecci KC, Santos BVO, Souto JT. The Bisindole Alkaloid Caulerpin, from Seaweeds of the Genus Caulerpa, Attenuated Colon Damage in Murine Colitis Model. Mar Drugs. 2018; 16:318. https://doi.org/10.3390/md16090318 PMID: $\underline{30205459}$

37. Leng L, Cao R, Ma J, Mou D, Zhu Y, Li W, Lv L, Gao D, Zhang S, Gong F, Zhao L, Qiu B, Xiang $H$, et al. Pathological features of COVID-19-associated lung injury: a preliminary proteomics report based on clinical samples. Signal Transduct Target Ther. 2020; 5:240. https://doi.org/10.1038/s41392-020-00355-9 PMID: $\underline{33060566}$

38. Grimes JM, Grimes KV. p38 MAPK inhibition: A promising therapeutic approach for COVID-19. J Mol Cell Cardiol. 2020; 144:63-65.

https://doi.org/10.1016/i.yimcc.2020.05.007 PMID: $\underline{32422320}$

39. Cheng $Y$, Sun F, Wang L, Gao M, Xie Y, Sun Y, Liu H, 
Yuan Y, Yi W, Huang Z, Yan H, Peng $K$, Wu Y, Cao Z. Virus-induced p38 MAPK activation facilitates viral infection. Theranostics. 2020; 10:12223-40.

https://doi.org/10.7150/thno.50992

PMID:33204339

40. Niu J, Ma Z, Nuerlan A, Li S, Cui W, Gao H, Abulajiang G, Zhang W, Li X. Prognostic value of MYD88 L265P mutation in diffuse large $B$ cell lymphoma via droplet digital PCR. Mol Med Rep. 2020; 22:1243-56. https://doi.org/10.3892/mmr.2020.11186 PMID: $\underline{32468019}$

41. Ha YJ, Hur J, Go DJ, Kang EH, Park JK, Lee EY, Shin K, Lee EB, Song YW, Lee YJ. Baseline peripheral blood neutrophil-to-lymphocyte ratio could predict survival in patients with adult polymyositis and dermatomyositis: A retrospective observational study. PLoS One. 2018; 13:e0190411. https://doi.org/10.1371/journal.pone.0190411 PMID:29293605

42. Bawage SS, Tiwari PM, Singh A, Dixit S, Pillai SR, Dennis VA, Singh SR. Gold nanorods inhibit respiratory syncytial virus by stimulating the innate immune response. Nanomedicine. 2016; 12:2299310. https://doi.org/10.1016/j.nano.2016.06.006 PMID:27381068

43. Wijnant SRA, Jacobs M, Van Eeckhoutte HP, Lapauw $B$, Joos GF, Bracke KR, Brusselle GG. Expression of
ACE2, the SARS-CoV-2 Receptor, in Lung Tissue of Patients With Type 2 Diabetes. Diabetes. 2020; 69:2691-99.

https://doi.org/10.2337/db20-0669

PMID:33024003

44. Menon R, Otto EA, Sealfon R, Nair V, Wong AK, Theesfeld CL, Chen X, Wang Y, Boppana AS, Luo J, Yang Y, Kasson PM, Schaub JA, et al. SARS-CoV-2 receptor networks in diabetic and COVID-19associated kidney disease. Kidney Int. 2020; 98:150218.

https://doi.org/10.1016/j.kint.2020.09.015 PMID:33038424

45. Introini A, Boström S, Bradley F, Gibbs A, Glaessgen A, Tjernlund A, Broliden K. Correction: Seminal plasma induces inflammation and enhances HIV-1 replication in human cervical tissue explants. PLoS Pathog. 2017; 13:e1006492.

https://doi.org/10.1371/journal.ppat.1006492

PMID:28700681

46. Lérias $S$, Esteves $S$, Silva $F$, Cunha $M$, Cochicho $D$, Martins L, Félix A. CD274 (PD-L1), CDKN2A (p16), TP53, and EGFR immunohistochemical profile in primary, recurrent and metastatic vulvar cancer. Mod Pathol. 2020; 33:893-904.

https://doi.org/10.1038/s41379-019-0429-z

PMID:31844270 


\section{SUPPLEMENTARY MATERIALS}

\section{Supplementary Figures}

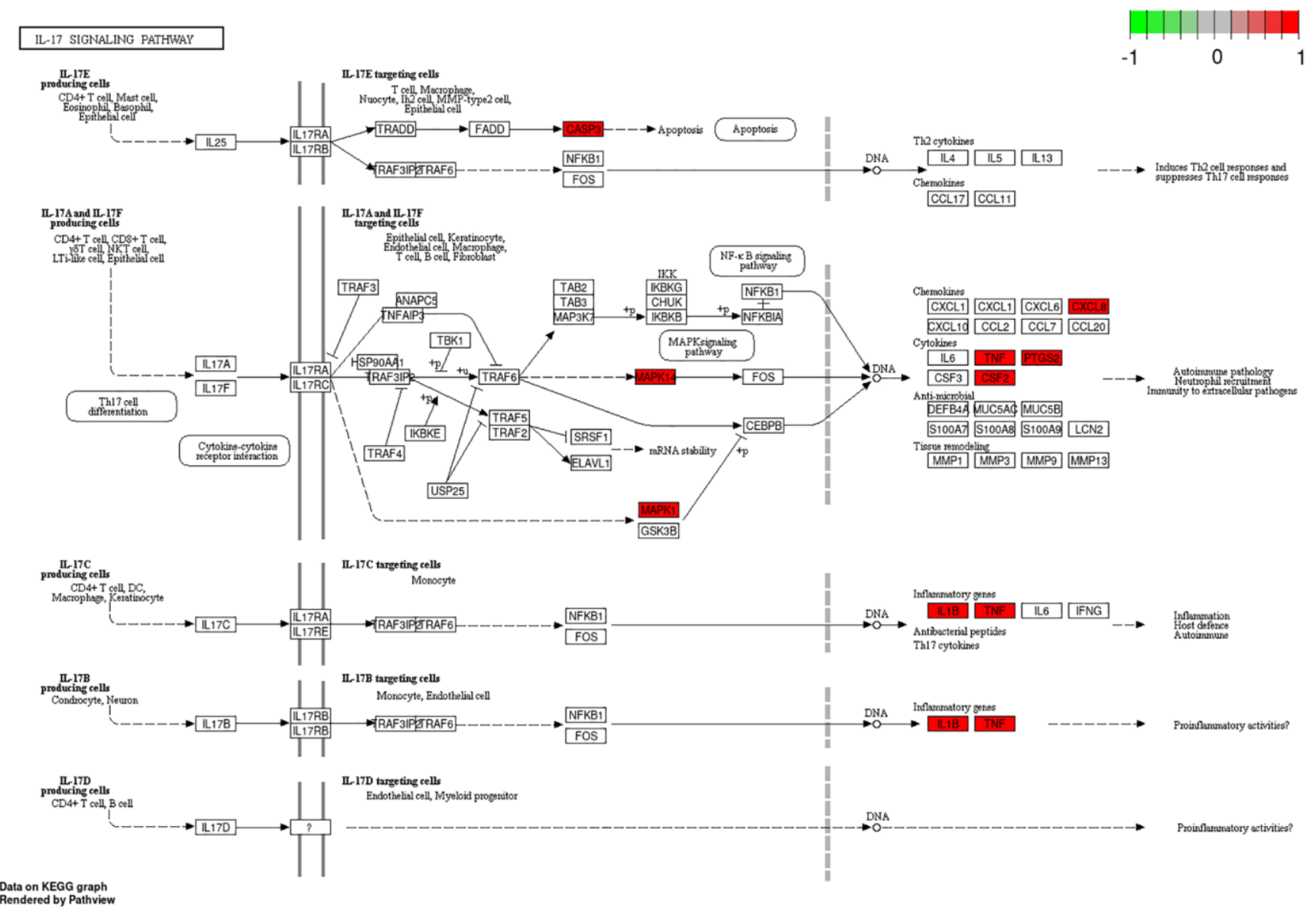

Supplementary Figure 1. IL-17 signaling pathway as one of the main pathways related to the therapeutic mechanism of emodin. 


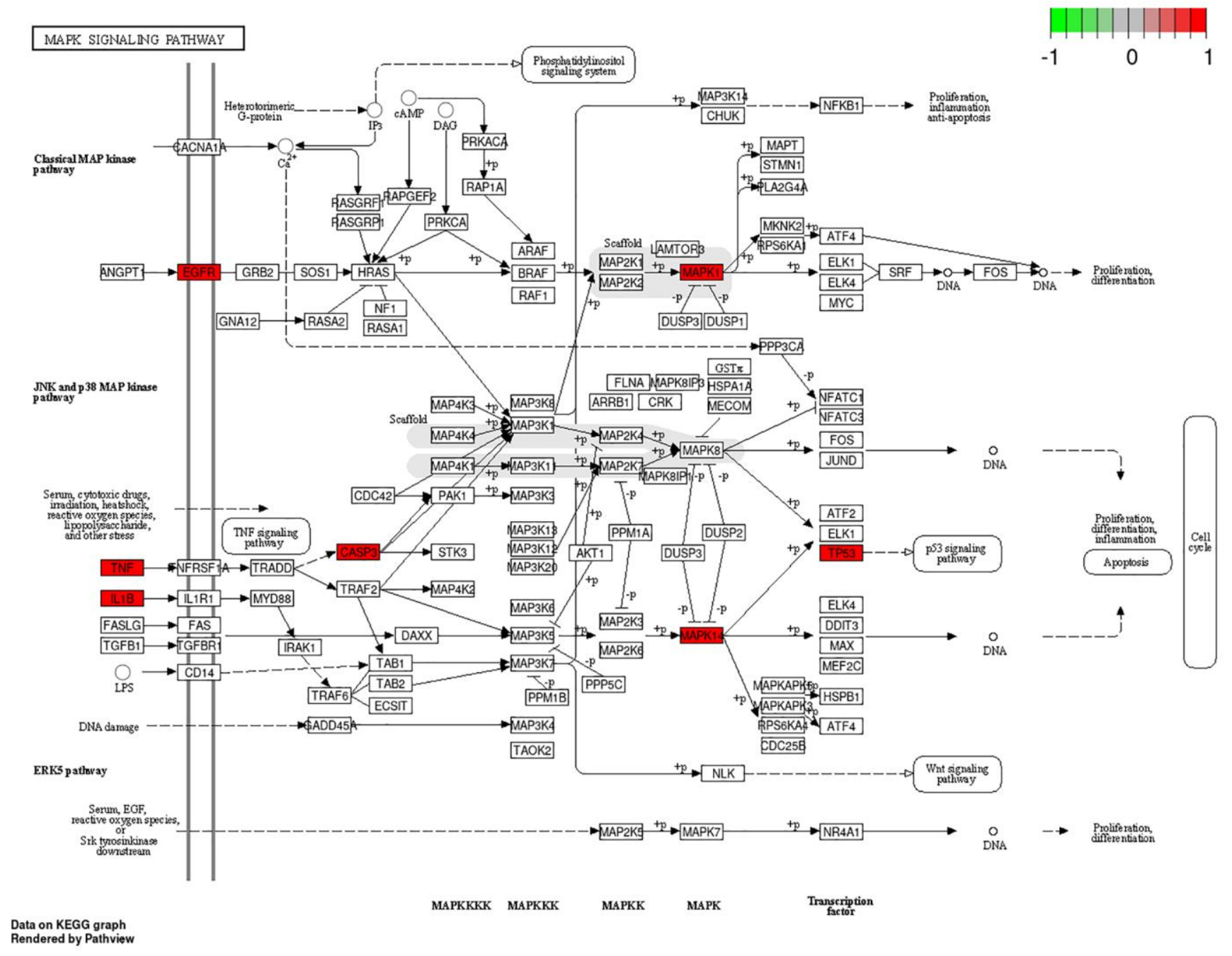

Supplementary Figure 2. MAPK signaling pathway as one of the main pathways related to the therapeutic mechanism of emodin. 


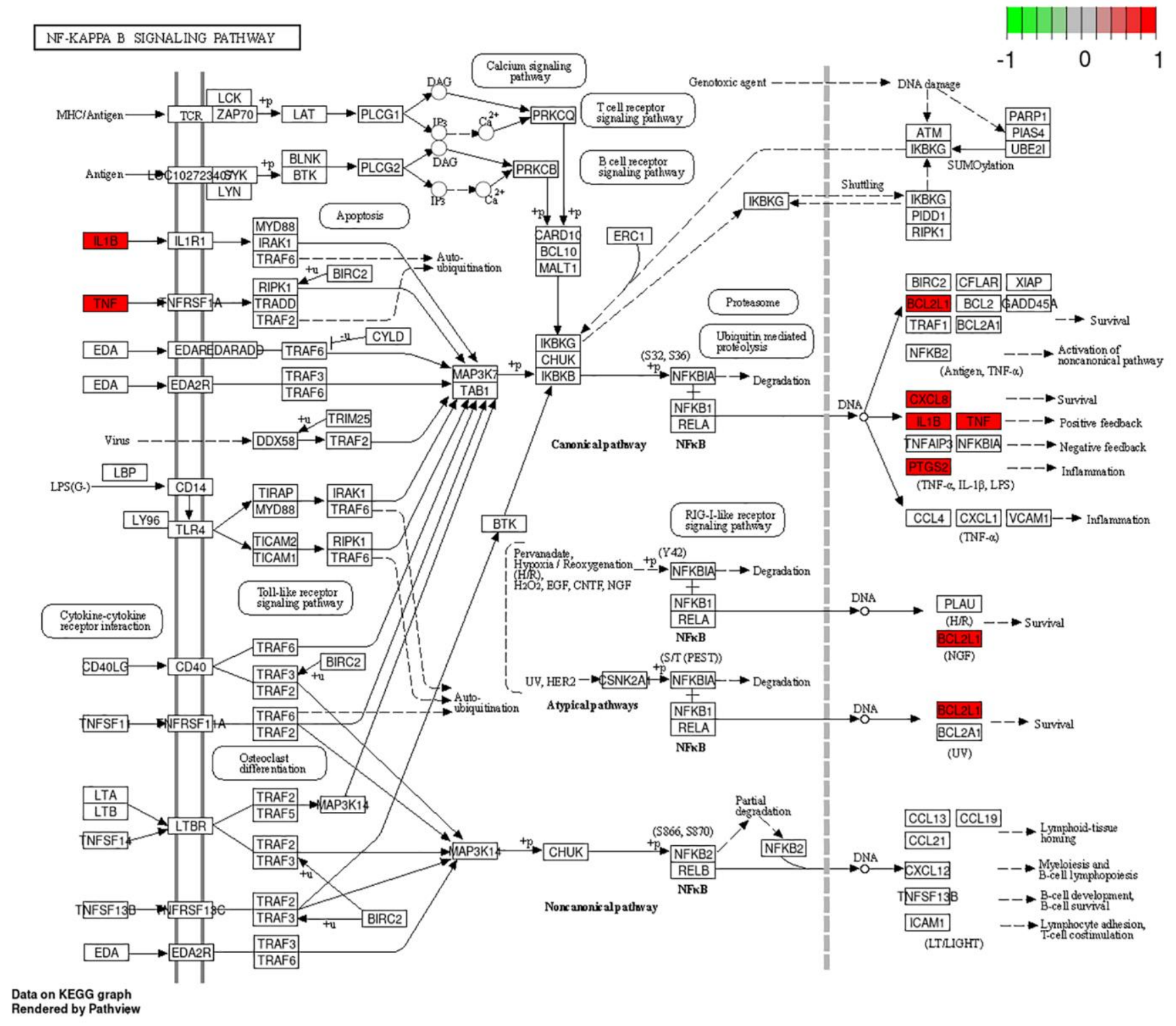

Supplementary Figure 3. NF-kappa B signaling pathway as one of the main pathways related to the therapeutic mechanism of emodin. 


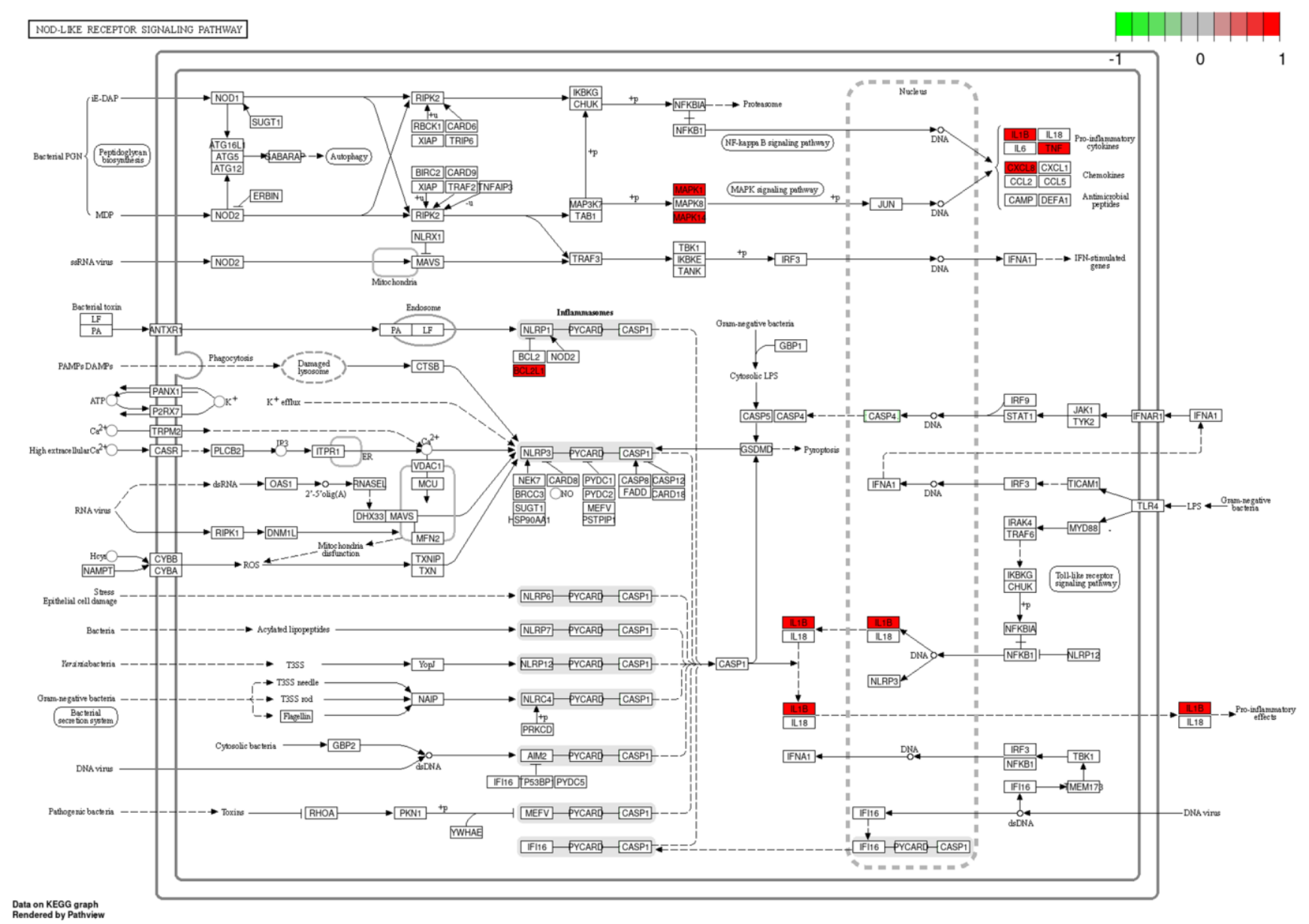

Supplementary Figure 4. NOD-like receptor signaling pathway as one of the main pathways related to the therapeutic mechanism of emodin. 

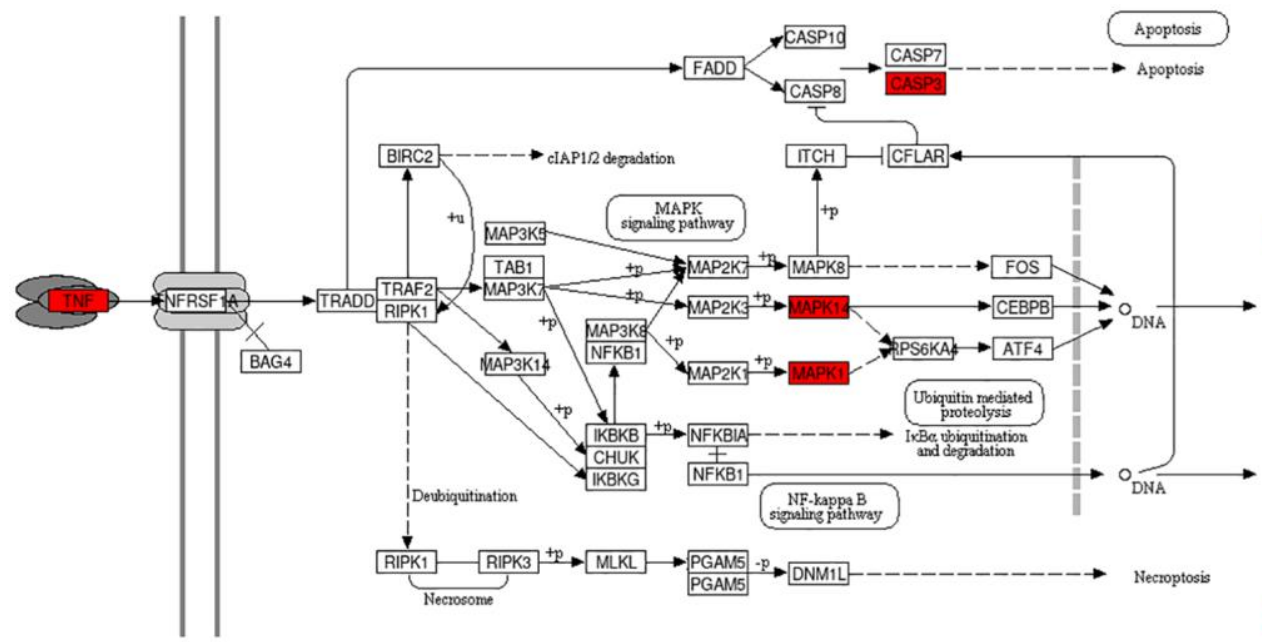

Loukocyte recruitrent

CCL2 2 CCL5 CCL20

\begin{tabular}{llll}
\hline CXCL1 & CXCL1 & CXCL \\
\hline
\end{tabular}

CXCL6 CXCL19 CX3CL1

Leukocyte activation

\begin{tabular}{l} 
Suffece recpptors \\
\hline FAS [L18R1 JAG1
\end{tabular}

influmnatory cytokines

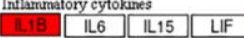

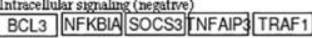

Intracelluhar signaling (positive)
[fif7]

\begin{tabular}{l} 
Transerintion fostors \\
\hline FOS JUN JUNB \\
\hline
\end{tabular}

Remodebingof extracelluhar mathix

Vascularefiects

\begin{tabular}{ll}
\hline PRRS \\
\hline NOD2 \\
\hline
\end{tabular}

Cell adhesion

Snthesis of inflarmatory mediatos

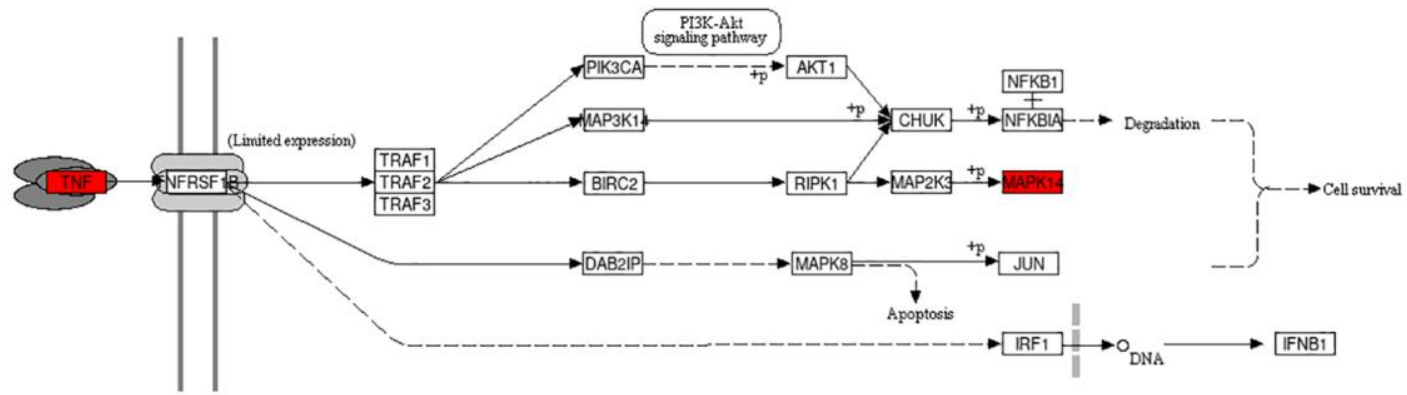

Data on KEGG graph

Rendered by Pathview

Supplementary Figure 5. TNF signaling pathway as one of the main pathways related to the therapeutic mechanism of emodin. 